PlastOx 2007 (2009) 277-286

(C) EDP Sciences, 2009

DOI: $10.1051 /$ ptox/2009020

\title{
Les siliciures de métaux de transition en microélectronique : propriétés mécaniques et contraintes induites au cours de la formation en phase solide
}

\author{
O. Thomas \\ Aix-Marseille Université, IM2NP et CNRS, IM2NP (UMR 6242), Faculté des Sciences et \\ Techniques, Campus de Saint-Jérôme, Avenue Escadrille Normandie Niemen, Case 262, \\ 13397 Marseille Cedex, France
}

\begin{abstract}
Résumé. Depuis le début des années 1980, des siliciures de métaux de transition ( $\left.\mathrm{WSi}_{2}, \mathrm{TiSi}_{2}, \mathrm{CoSi}_{2}, \mathrm{NiSi}\right)$ sont utilisés comme matériaux de contact et d'interconnection dans les dispositifs de la microélectronique. Ils sont formés par diffusion réactive entre un film mince de métal et le silicium. Des contraintes importantes se développent lors de la formation de la nouvelle phase intermétallique. Ces contraintes peuvent être analysées expérimentalement par mesures de courbure et diffraction des rayons $\mathrm{X}$ in situ. Les études expérimentales montrent que les paramètres importants qui contrôlent le développement de ces contraintes sont : (i) la variation de volume molaire. (ii) la cinétique de croissance. (iii) le mécanisme de relaxation des contraintes. Les modèles existants permettent une description phénoménologique de l'évolution des contraintes mais une compréhension physique plus fine nécessite la connaissance des propriétés mécaniques des couches très minces de siliciures.
\end{abstract}

\section{UNE BRÈVE HISTOIRE DES SILICIURES : DU FOUR ÉLECTRIQUE À LA MICROÉLECTRONIQUE}

La plupart des métaux de transition forment des composés intermétalliques avec le silicium. La cristallographie de ces composés [1,2] est en général assez bien connue mais leurs propriétés physiques le sont moins. Le disiliciure de $\mathrm{Mo}, \mathrm{MoSi}_{2}$, a été utilisé très tôt comme une protection contre l'oxydation de conducteurs en Mo. Alors que le molybdène pur est très sensible à l'oxydation à cause de la formation d'oxydes volatils, $\mathrm{MoSi}_{2}$ s'oxyde en formant une couche protectrice de $\mathrm{SiO}_{2}$. Ainsi Henri Moissan, prix Nobel de Chimie en 1906 et inventeur du four électrique a-t-il proposé de former une couche protectrice de siliciure par réaction en phase solide entre un barreau de Mo et de la poudre de Si. Ces mêmes propriétés de $\mathrm{MoSi}_{2}$, à savoir caractère réfractaire et résistance à l'oxydation, sont utilisées encore aujourd'hui dans les enroulements de fours électriques dits SuperKanthal ${ }^{\circledR}$.

L'utilisation des siliciures de métaux de transition en microélectronique est beaucoup plus récente. Depuis l'invention de la technologie planar par R. Noyce et J. Kilby [3] en 1959 la microélectronique a réussi à maintenir un rythme effréné de miniaturisation [4]. La figure de mérite souvent utilisée pour caractériser une génération de circuits est la longueur du canal d'un transistor élémentaire. Nous sommes aujourd'hui (début 2008) au croisement entre la génération $65 \mathrm{~nm}$ et la génération $45 \mathrm{~nm}$ ! Cette miniaturisation passe de plus en plus par l'introduction de nouveaux matériaux : diélectriques à faible permittivité pour les interconnexions, diélectriques à forte permittivité pour les oxydes de grille, ... Ainsi la microélectronique silicium est devenue un terrain de jeu extraordinaire pour la science des matériaux. Les siliciures de métaux de transition sont apparus au début des années 1980 dans les circuits [5]. Ils permettent de diminuer les résistances d'accès au niveau de la source et du drain des transistors et aussi permettent de shunter la résistance du silicium polycristallin de grille. En effet un nombre important de siliciures sont en fait de bons métaux avec des résistivités à la température ambiante de l'ordre de 10 à $20 \mu \Omega \mathrm{cm}$ [6]. Les premiers siliciures utilisés furent des siliciures de métaux réfractaires [5] comme $\mathrm{WSi}_{2}$ à cause de leur bonne stabilité thermique. Avec la diminution des dimensions il a été remplacé par $\mathrm{TiSi}_{2}$ qui se forme à plus basse température. Ce dernier du être 
remplacé par la suite par $\mathrm{CoSi}_{2}$ car il posait des problèmes de nucléation dans les lignes étroites. Enfin, pour les générations de circuits les plus avancées c'est NiSi qui a été choisi à cause de sa faible résistivité, sa formation à basse température et la faible consommation de silicium lors de sa formation. Quelques propriétés importantes des siliciures pertinents pour la microélectronique sont données dans le tableau 1.

Tableau 1. Quelques caractéristiques de siliciures utilisés en microélectronique [7].

\begin{tabular}{|c|c|c|c|c|c|c|}
\hline Siliciure & $\begin{array}{c}\text { T de } \\
\text { formation } \\
\left({ }^{\circ} \mathbf{C}\right)\end{array}$ & $\begin{array}{c}\text { T de } \\
\text { fusion } \\
\left({ }^{\circ} \mathbf{C}\right)\end{array}$ & $\begin{array}{c}\text { Unité de Si } \\
\text { consommé par } \\
\text { unité de métal }\end{array}$ & $\begin{array}{c}\text { Unité de } \\
\text { siliciure } \\
\text { formé par } \\
\text { unité de métal }\end{array}$ & $\begin{array}{c}\text { Espéce } \\
\text { diffusante } \\
\text { majoritaire }\end{array}$ & $\begin{array}{c}\text { Résistivité } \\
\text { Résistivité } \\
(\mu \Omega \cdot \mathbf{c m})\end{array}$ \\
\hline $\mathbf{T i S i}_{2} \mathbf{C 4 9}$ & $700-900$ & & 2,22 & 2,33 & $\mathrm{Si}$ & 80 \\
\hline $\mathbf{T i S i}_{2} \mathbf{C 5 4}$ & $700-900$ & 1500 & 2,22 & 2,44 & $\mathrm{Si}$ & $13-16$ \\
\hline $\mathbf{C o}_{2} \mathbf{S i}$ & $300-450$ & 1330 & 0,90 & 1,47 & $\mathrm{Co}$ & 70 \\
\hline $\mathbf{C o S i}$ & $450-650$ & 1460 & 1,81 & 1,98 & $\mathrm{Si}$ & 147 \\
\hline $\mathbf{C o S i}_{2}$ & $650-900$ & 1330 & 3,61 & 3,49 & $\mathrm{Co}$ & 15 \\
\hline $\mathbf{N i}_{2} \mathbf{S i}$ & $200-350$ & 1300 & 0,91 & 1,49 & $\mathrm{Ni}$ & 24 \\
\hline $\mathbf{N i S i}$ & $350-750$ & 900 & 1,83 & 2,01 & $\mathrm{Ni}$ & $10-20$ \\
\hline $\mathbf{N i S i}_{2}$ & $750-900$ & 993 & 3,66 & 3,59 & $\mathrm{Ni}$ & $40-50$ \\
\hline $\mathbf{M o S i}_{2}$ & 525 & 2020 & 2,57 & 2,60 & $\mathrm{Si}$ & $12-20$ \\
\hline $\mathbf{T a S i}_{2}$ & 650 & 2200 & 2,21 & 2,40 & $\mathrm{Si}$ & $20-40$ \\
\hline $\mathbf{W S i} \mathbf{m}_{2}$ & 650 & 2160 & 2,52 & 2,58 & $\mathrm{Si}$ & 13 \\
\hline
\end{tabular}

La figure 1 est une représentation schématique du procédé «salicide» (pour self-aligned silicide) utilisé pour métalliser par siliciuration la grille, la source et le drain des transistors MOS. Le procédé en trois étapes permet de siliciurer en même temps les trois zones et ce de façon alignée. La justification du procédé en deux recuits était semble t-il liée au risque de court circuit lorsque le siliciure final désiré se forme par diffusion du silicium.

\section{FORMATION D'UN SILICIURE PAR RÉACTION EN PHASE SOLIDE EN FILM MINCE : CINÉTIQUE DE CROISSANCE ET DÉVELOPPEMENT DES CONTRAINTES}

Comme on peut le voir sur la figure 1 les siliciures sont - en microélectronique - formés par réaction en phase solide entre un film mince de métal préalablement déposé et le silicium poly- ou mono-cristallin. Les mécanismes de formation en films minces des siliciures ont été très étudiés [8-10]. En particulier d'Heurle [9] a clairement mis en évidence que lorsque la force motrice de la réaction est suffisamment faible la cinétique de formation est contrôlée par l'étape de nucléation. La morphologie qui en résulte est en général assez rugueuse. Lorsque ce n'est pas le cas il a été bien souvent observé une croissance contrôlée par la diffusion avec une formation séquentielle [10], contrairement à ce qui est observé dans les couples de diffusion massifs. L'apparition des alliages $\mathrm{Si}_{\mathrm{x}} \mathrm{Ge}_{1-\mathrm{x}}$ en microélectronique dés les années 1986-87 a bien évidemment suscité des études sur la formation de silico-germaniures par diffusion réactive [11].

La formation d'un siliciure par diffusion réactive en phase solide donne lieu à une variation de volume considérable. Les contraintes qui en résulte [12] ont été comparativement beaucoup moins étudiées que les cinétiques de croissance. Les tendances récentes de la microélectronique, qui visent à utiliser les contraintes mécaniques pour modifier la mobilité des porteurs de charge dans le canal des transistors [13], redonnent de l'actualité à ce genre d'études. L'apparition de contraintes générées par la formation d'une solution solide ou bien d'une nouvelle phase est un problème général en métallurgie. Par exemple, les contraintes générées lors de l'oxydation des métaux [14] peuvent provoquer le décollement de la couche d'oxyde. La modélisation quantitative des contraintes générées lors de l'interdiffusion reste un problème extrèmement difficile. Il est nécessaire de prendre en compte le couplage [15-17] entre la cinétique, la thermodynamique et la mécanique. D’autre part la relaxation 

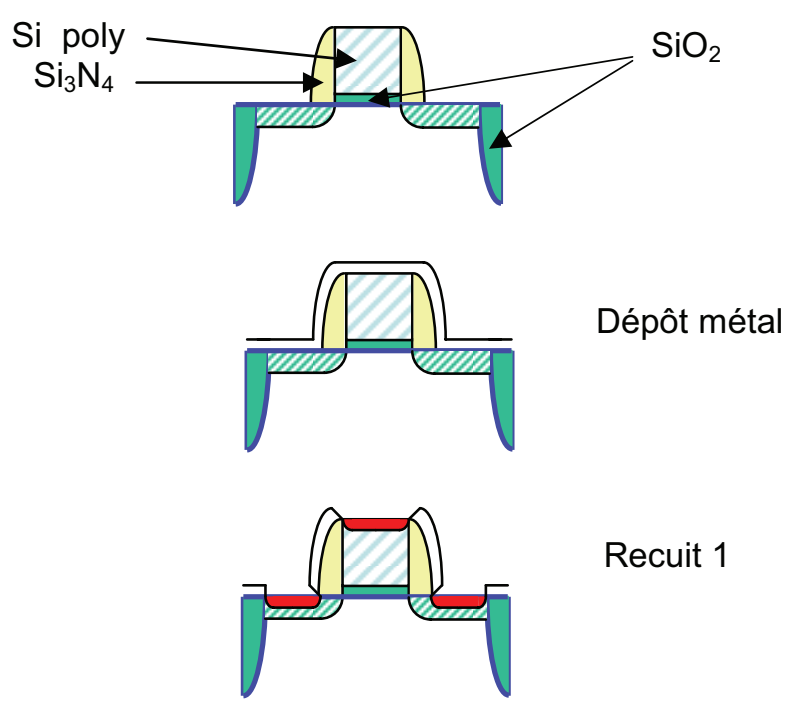

Recuit 1

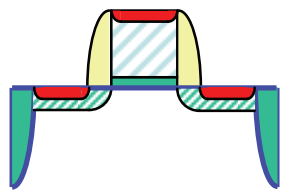

Retrait métal

+ Recuit 2

Figure 1. Représentation schématique du procédé « salicide».

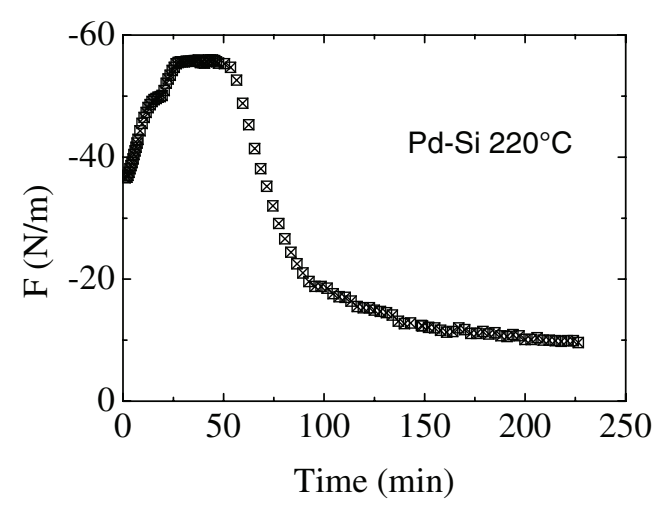

(a)

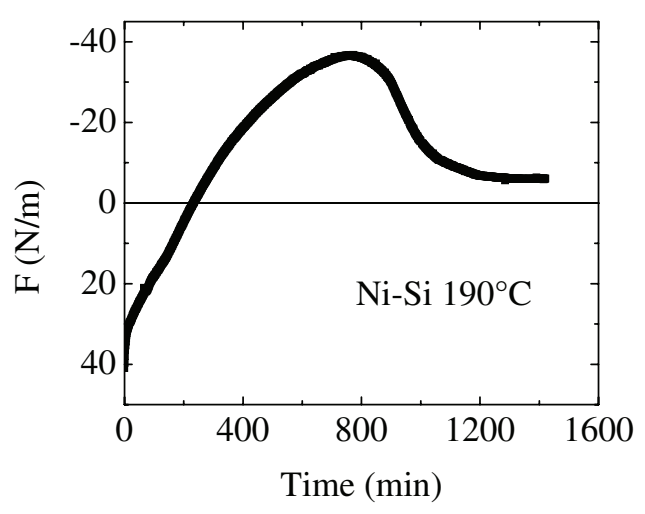

(b)

Figure 2. Mesures de courbure au cours de la réaction de films minces de Pd et de $\mathrm{Ni}$ avec $\mathrm{Si}(001)$. (a) force pendant la réaction de $100 \mathrm{~nm}$ de $\mathrm{Pd}$ avec $\mathrm{Si}(001)$ à $220^{\circ} \mathrm{C}$; (b) force pendant la réaction de $18 \mathrm{~nm}$ de $\mathrm{Ni}$ avec Si $(001)$ à $190^{\circ} \mathrm{C}$.

des contraintes qui se produit en cours de croissance doit être également considérée. La relaxation des contraintes dans les couches minces et les structures de petites dimensions [18-20] est un sujet de recherche actif. Il est désormais bien établi que la limite élastique dépend de la taille.

D'un point de vue expérimental la mesure de la courbure du substrat [21, 22] est une méthode très versatile qui permet de déterminer la force appliquée par le dépôt. Deux exemples de mesures in situ de la force par unité de largeur au cours de la formation de $\mathrm{Pd}_{2} \mathrm{Si}$ (Fig. 2a) et de $\mathrm{Ni}_{2} \mathrm{Si}$ (Fig. 2b) 
par réaction entre un film de $\mathrm{Pd}$ ou de $\mathrm{Ni}$ avec $\mathrm{Si}$ (001) sont présentés en figure 2. Dans les deux cas le siliciure qui se forme est le premier de la séquence. Dans le cas du $\mathrm{Ni}$, toutefois, la présence de phases riches en métal ont parfois été signalées. Les deux courbes de la figure 2 partagent un certain nombre de caractéristiques communes. Dans les deux cas la force commence par évoluer en compression avant de relaxer progressivement vers zéro. Ceci montre que le siliciure formé est libre de contrainte à condition d'attendre suffisamment longtemps à la température de formation. Si ces conditions sont réunies la contrainte résiduelle dans le siliciure à la température ambiante ne reflète plus la contrainte de croissance mais simplement le comportement thermo-mécanique du film de siliciure au cours du refroidissement.

La variation de volume au cours de la formation d'une nouvelle phase est considérable. C'est en général cette variation de volume qui est considérée comme la cause des contraintes qui se développent. Dans le cas de l'oxydation il est habituel de se reporter au rapport de Pillings-Bedworth entre le volume de métal et le volume de l'oxyde. Pour une couche mince supportée par un substrat rigide, le changement de volume est nécessairement accommodé par une déformation dans le plan :

$$
\varepsilon_{/ /}=-\frac{1}{3} \frac{\Delta \mathrm{V}}{\mathrm{V}}
$$

où $\varepsilon_{/ /}$est la deformation dans le plan et $\Delta \mathrm{V} / \mathrm{V}$ le changement de volume correspondant à l'apparition de la nouvelle phase. Dans le cas de la précipitation de $\mathrm{A}_{\mathrm{x}} \mathrm{B}_{\mathrm{y}}$ à partir d'un alliage homogène, le changement de volume à prendre en compte est

$$
\frac{\Delta \mathrm{V}}{\mathrm{V}}=\frac{\mathrm{V}_{\mathrm{A}_{\mathrm{x}} \mathrm{B}_{\mathrm{y}}}-\mathrm{xV}_{\mathrm{A}}-\mathrm{yV} \mathrm{V}_{\mathrm{B}}}{\mathrm{xV}_{\mathrm{A}}+\mathrm{y} \mathrm{V}_{\mathrm{B}}}
$$

En général $\Delta \mathrm{V} / \mathrm{V}$ est négatif car le compose ordonné est plus dense que l'alliage désordonné. Il s'ensuit l'apparition d'une contrainte en tension dans le précipité. Dans le cas d'une diffusion réactive en couche mince le problème est toutefois différent puisque la réaction se produit à une seule interface. Dans le cas de la croissance de $\mathrm{Ni}_{2} \mathrm{Si}$ par reaction entre un film de $\mathrm{Ni}$ et le $\mathrm{Si}$, la réaction se produit à l'interface $\mathrm{Ni}_{2} \mathrm{Si} / \mathrm{Si}$ car c'est le Ni qui diffuse plus vite [23] que le $\mathrm{Si}$ dans $\mathrm{Ni}_{2} \mathrm{Si}$. La variation de volume à prendre en compte est donc

$$
\frac{\Delta \mathrm{V}}{\mathrm{V}}=\frac{\mathrm{V}_{\mathrm{Ni}_{2} \mathrm{Si}}-\mathrm{V}_{\mathrm{Si}}}{\mathrm{V}_{\mathrm{Si}}}
$$

qui est positive. On s'attend donc à une évolution de la contrainte en compression, ce qui est en accord avec les observations. Le même raisonnement peut être fait pour de nombreux siliciures [12] indépendamment de la nature (métal ou silicium) de l'espèce qui diffuse le plus rapidement. Dans le cas $\left(\mathrm{MoSi}_{2}\right.$ par exemple) où le silicium est l'espèce qui diffuse le plus vite, la contrainte sera liée à la difference de volume entre le metal et le siliciure. Dans la plupart des cas on prédit des contraintes compressives en accord avec les constatations expérimentales.

Une description quantitative des contraintes qui apparaissent lors de la siliciuration est beaucoup plus difficile. La variation de volume déduite de l'équation (3) est énorme : dans le cas de la croissance de $\mathrm{Ni}_{2} \mathrm{Si}$ elle vaut $66 \%$ ! Ceci conduirait à une contrainte biaxiale largement supérieure à la cission critique. Expérimentalement la contrainte initiale dans le siliciure est de l'ordre de $-2 \mathrm{GPa}$. En supposant que la variation de volume de $66 \%$ est accommodée élastiquement et plastiquement, l'énergie mécanique totale est

$$
\mathrm{W}_{\mathrm{def}}=\frac{1-v}{\mathrm{E}} \sigma^{2}+\sigma\left(-\frac{1}{3} \frac{\Delta \mathrm{V}}{\mathrm{V}}-\frac{1-v}{\mathrm{E}} \sigma\right)=-\frac{1}{3} \frac{\Delta \mathrm{V}}{\mathrm{V}} \sigma
$$

où $\mathrm{E}$ et $v$ sont le module d'Young et le coefficient de Poisson du siliciure. Cette énergie de déformation est de l'ordre de $0.44 \mathrm{~kJ} / \mathrm{cm}^{3}$, soit environ $30 \mathrm{meV}$. Une telle énergie reste faible mais n'est pas négligeable en comparaison de l'énergie de formation de $\mathrm{Ni}_{2} \mathrm{Si}$ [24], qui vaut $7.6 \mathrm{~kJ} / \mathrm{cm}^{3}$. On peut donc $\mathrm{s}$ 'attendre à un couplage complexe entre les contraintes et la cinétique de croissance. 


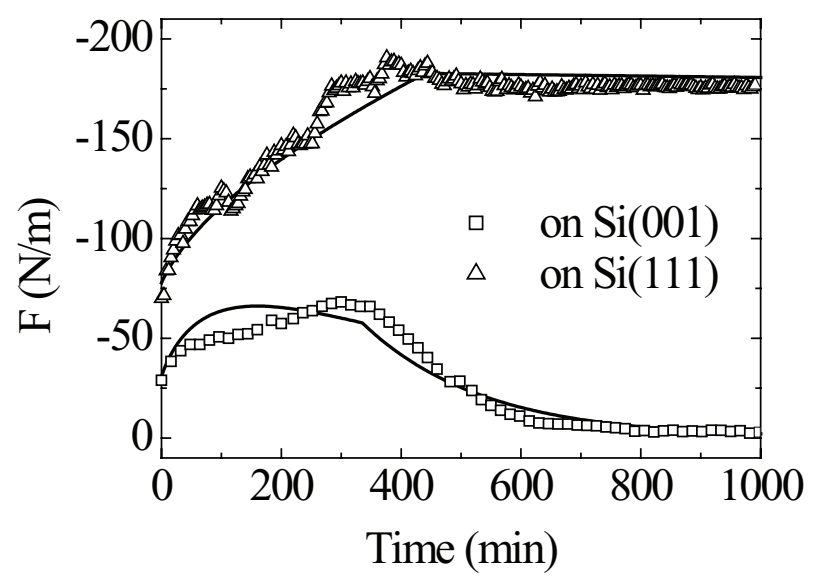

Figure 3. Mesures de courbure in situ à $220^{\circ}$ pendant la réaction de films minces de $\mathrm{Pd}$ avec Si (001) et Si (111). Les courbes continues correspondent au modèle de Zhang et d'Heurle.

Le mécanisme de relaxation de la contrainte est le deuxième élément nécessaire à la description de l'évolution des contraintes dans la couche de siliciure en croissance. Comme on peut le voir dans la figure 2, une fois la croissance du siliciure terminée c'est la relaxation de la contrainte qui domine. Au cours de la croissance on peut donc s'attendre à ce que l'établissement de la contrainte et la relaxation se produisent de façon simultanée. Le mécanisme de relaxation de la contrainte est un problème complexe et ce pour au moins deux raisons fondamentales : (i) il dépend de la microstructure qui elle même évolue au cours de la croissance du film. (ii) les mécanismes de relaxation des contraintes dans les couches minces sont différents [18-20] de ce qu'ils sont dans les matériaux massifs. La limite élastique des films minces dépend étroitement de leur épaisseur. Un exemple frappant de l'influence de la microstructure du film de siliciure sur la relaxation et par conséquent sur l'évolution de la contrainte est montré en figure 3. Le siliciure de $\mathrm{Pd}, \mathrm{Pd}_{2} \mathrm{Si}$, est hexagonal et s'épitaxie sur $\mathrm{Si}$ (111) avec la relation d'épitaxie suivante : (00.1) [110] $\mathrm{Pd}_{2} \mathrm{Si} / /$ (111) [110] Si. Sur Si (001), $\mathrm{Pd}_{2} \mathrm{Si}$ est polycristallin avec une texture de fibre (00.1). Il en résulte une différence de mosaïcité autour de l'axe [001] de $\mathrm{Pd}_{2} \mathrm{Si}$ [25] considérable : $18^{\circ}$ sur $\mathrm{Si}(001)$ et $1.8^{\circ}$ sur Si (111). Comme le montre la figure 3 , la relaxation de la contrainte est beaucoup plus rapide [25] sur Si (001) que sur Si (111). Comme on pouvait s'y attendre la relaxation est plus difficile dans le film monocristallin que dans le film polycristallin. Cette différence de comportement peut s'expliquer de plusieurs manières. Si l'on suppose que la relaxation dans le siliciure s'effectue par un mécanisme de glissement de dislocations et que d'autre part les plans de glissement sont les plans basaux (00.1), alors le film épitaxié se trouve dans une situation telle que la contrainte résolue sur ces plans est nulle. En revanche dans le cas du siliciure sur Si (001) la mosaïcité importante (presque $20^{\circ}$ ) assure l'existence d'une contrainte résolue non nulle dans les grains désorientés. Ce seront donc de tels grains qui pourront relaxer plastiquement. La différence de relaxation entre $\mathrm{Pd}_{2} \mathrm{Si}$ / $\mathrm{Si}(111)$ et $\mathrm{Pd}_{2} \mathrm{Si} / \mathrm{Si}(001)$ peut également s'expliquer dans le cas où la relaxation s'effectuerait par fluage diffusionnel. En effet un fluage par diffusion intergranulaire (Coble) [26] devrait être beaucoup plus efficace dans le cas du film polycristallin $\mathrm{Pd}_{2} \mathrm{Si} / \mathrm{Si}(001)$ que dans le cas du film épitaxié $\mathrm{Pd}_{2} \mathrm{Si}_{\text {/ }}$ $\operatorname{Si}(111)$.

A partir de la compétition croissance - relaxation observée expérimentalement, Zhang et d'Heurle [27] ont proposé un modèle phénoménologique qui décrit le comportement de la force $F(t)$ au cours de la réaction en phase solide. Le modèle décrit la variation $\mathrm{F}(\mathrm{t})$ comme résultant d'une compétition entre l'établissement de la contrainte liée à la formation de la nouvelle phase et la relaxation de cette contrainte. Le premier terme dépend de la cinétique de croissance du siliciure alors que le second dépend du mécanisme de relaxation. Un mécanisme de fluage newtonien avec un temps de relaxation $\tau$ a été supposé. 
La contrainte à la profondeur z (définie à partir de l'interface de croissance) et à l'instant $\mathrm{t}$ dans la couche de siliciure d'épaisseur $\mathrm{dz}$ est la contrainte de croissance $\sigma_{0}$, affectée d'une décroissance exponentielle due à la relaxation pendant un temps lié au moment où cette tranche $\mathrm{dz}$ s'est formée.

$$
\sigma(\mathrm{z}, \mathrm{t})=\sigma_{0} \exp \left(-\frac{\mathrm{t}-\mathrm{h}^{-1}(\mathrm{z})}{\tau}\right)
$$

$\mathrm{z}=\mathrm{h}(\mathrm{t})$ est la loi de croissance de la couche de siliciure. Dans le cas d'une croissance contrôlée par la diffusion on a une variation parabolique de l'épaisseur formée :

$$
h(t)=\sqrt{D t} \quad \text { ou } \quad h^{-1}(z)=\frac{z^{2}}{D} .
$$

La force $\mathrm{F}(\mathrm{t})$ s'obtient par intégration de $\sigma(\mathrm{z}, \mathrm{t})$ de $\mathrm{z}=0$ à $\mathrm{z}=\mathrm{h}(\mathrm{t})$. Ce modèle simple dépend de 3 paramètres, dont 2 peuvent être déterminés expérimentalement de manière directe. Le taux de croissance peut être mesuré indépendamment à partir de mesures d'intensité diffractée (ou bien par rétrodiffusion Rutherford, profilomètrie Auger, etc). Quant au temps de relaxation $\tau$ il peut être extrait de $\mathrm{F}(\mathrm{t})$ puisque celle-ci est proportionelle à $\exp (-\mathrm{t} / \tau)$ lorsque la croissance est terminée. On trouve dans la figure 3 des courbes $\mathrm{F}(\mathrm{t})$ calculées avec des paramètres consistants avec la croissance de $\mathrm{Pd}_{2} \mathrm{Si}$ à $220^{\circ} \mathrm{C}$ : $\mathrm{D}=1 \times 10^{-14} \mathrm{~cm}^{2} / \mathrm{s}, \tau=1.2 \times 10^{4} \mathrm{~s}, \sigma_{0}=-1.15 \mathrm{GPa}$ sur Si $(001)$ et $\mathrm{D}=7.3 \times 10^{-15} \mathrm{~cm}^{2} / \mathrm{s}, \tau=$ $3 \times 10^{6} \mathrm{~s}, \sigma_{0}=-1.2 \mathrm{GPa}$ sur $\mathrm{Si}(111)$. La comparaison avec les données expérimentales indique un accord raisonnable.

L'expression (5) correspond à l'intégration de l'équation différentielle qui décrit un écoulement visqueux à température constante. Dans le cas d'une expérience à température variable elle n'est plus valable. Cacho et Cailletaud [28] ont développé un modèle dans lequel la relaxation est décrite par une loi de Norton généralisée. La résolution numérique d'un tel modèle à partir des équations différentielles décrivant la mécanique du système permet de décrire l'évolution de la force même lorsque la température dépend du temps.

\section{PROPRIÉTÉS MÉCANIQUES DES SILICIURES : ÉLASTICITÉ ET PLASTICITÉ}

Contrairement aux propriétés électroniques [6] les propriétés mécaniques des siliciures de métaux de transition sont pour une large part très mal connues. Les propriétés élastiques ont seulement été déterminées pour quelques siliciures particuliers. Les constantes élastiques de quelques siliciures cubiques [29-31] sont données dans le tableau 2. A titre de comparaison les constantes élastiques du Si [32] et du $\mathrm{Cu}$ [33] sont également reportées. La figure 4 représente le module d'Young de $\mathrm{CoSi}_{2}$, qui a un facteur d'anisotropie élastique de 1,9, en fonction de la direction cristallographique.

Tableau 2. Constantes élastiques de quelques siliciures cubiques comparées à $\mathrm{Si}$ et à $\mathrm{Cu}$. $\mathrm{A}$ est le facteur d'anisotropie élastique.

\begin{tabular}{|l|l|l|l|l|l|}
\hline Composé & $\mathbf{C}_{11}(\mathbf{G P a})$ & $\mathbf{C}_{12}(\mathbf{G P a})$ & $\mathbf{C}_{44}(\mathbf{G P a})$ & $\mathbf{A}$ & Référence \\
\hline $\mathbf{S i}$ & 165,7 & 63,9 & 79,56 & 1,6 & 32 \\
\hline & & & & & \\
\hline $\mathbf{C o s i}_{\mathbf{2}}$ & 228 & 140 & 83 & 1,9 & 29 \\
\hline $\mathbf{N i S i}_{\mathbf{2}}$ & 199 & 141 & 53 & 1,8 & 30 \\
\hline $\mathbf{C o S i}$ & 328 & 95 & 120 & 1,0 & 31 \\
\hline & & & & & \\
\hline $\mathbf{C u}$ & 168,4 & 121,4 & 75,4 & 3,2 & 33 \\
\hline
\end{tabular}

Le tableau 2 ne fait pas apparaitre de comportement élastique très particulier pour $\mathrm{CoSi}_{2}, \mathrm{NiSi}_{2}$ ou bien CoSi. Ceci ne préjuge en rien des propriétés d'autres siliciures. Un siliciure tel que NiSi, très étudié en ce moment pour la microélectronique, reste encore très mal connu. NiSi est orthorhombique (structure type $\mathrm{MnP}$ ) et a un coefficient de dilatation $[34,35]$ très anisotrope : un de ses trois coefficients 


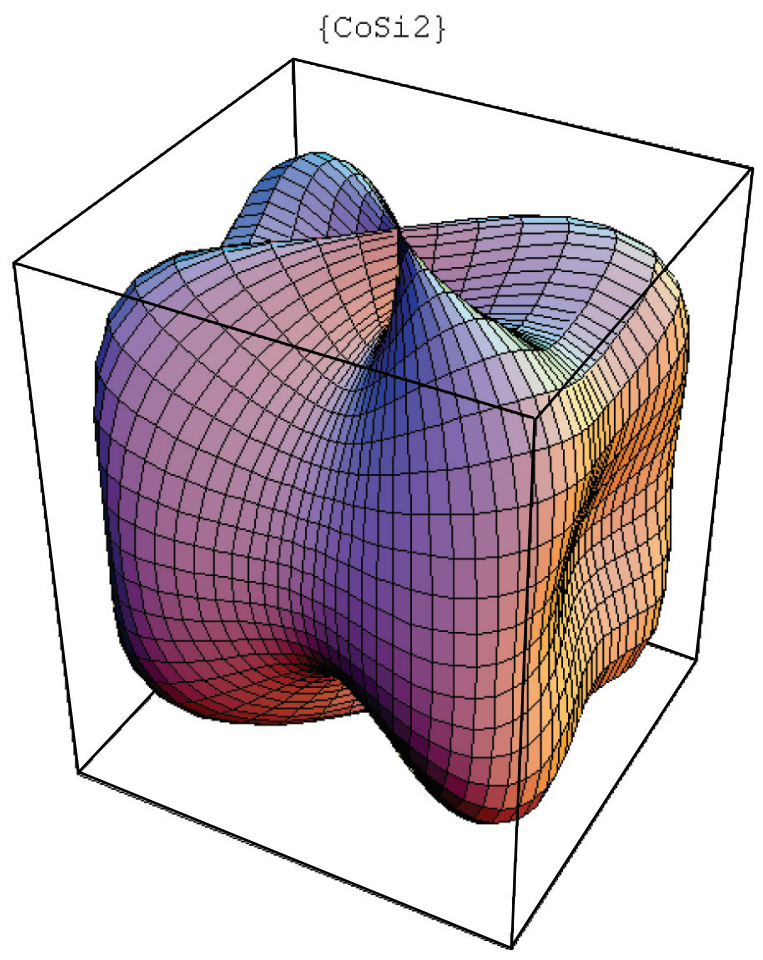

Figure 4. Module d'Young de $\mathrm{CoSi}_{2}$ en fonction de la direction cristallographique. Les $\mathrm{C}_{\mathrm{ij}}$ sont tirées de [29].
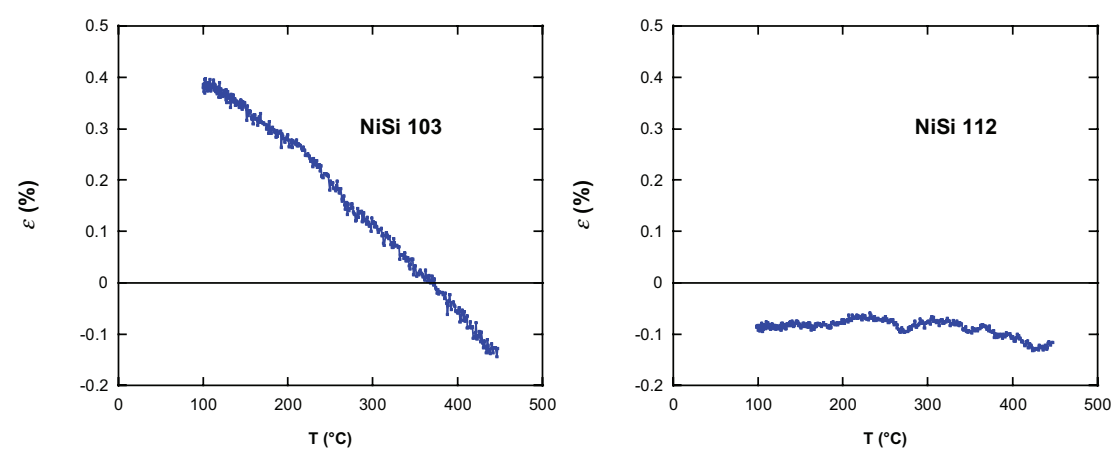

Figure 5. Déformations perpendiculaires dans un film de NiSi en fonction de la température. On observe une différence importante en fonction de l'orientation des grains.

de dilatation principaux, suivant l'axe [010], est même négatif ! Les propriétés élastiques de NiSi sont elles aussi «anormales» que sa dilatation thermique ? Des calculs sont en cours [36] pour répondre à cette question, importante pour décrire correctement les contraintes dans les films de NiSi. En tout état de cause on peut s'attendre à des variations considérables de contraintes de grain à grain dans un film polycristallin de NiSi. La figure 5 présente des mesures de déformation perpendiculaire (au plan de la couche) $[37,38]$ en fonction de la température dans deux familles de grains d'orientation différente dans un film polycristallin de NiSi. Le comportement de ces deux familles de grains est très différent. Il en résulte probablement des contraintes locales dans le silicium sous-jacent très différentes suivant que l'on se trouve sous une orientation du siliciure ou bien sous une autre. 
Alors qu'ils se comportent comme des métaux vis-à-vis du transport électronique, les siliciures sont en revanche fragiles à la température ambiante. Ce comportement mécanique est probablement à mettre en relation - au moins pour ce qui concerne les siliciures riches en silicium - avec le caractère partiellement covalent [39] de la liaison. Il en résulte une faible mobilité des dislocations dans les siliciures tant que la température n'est pas suffisamment élevée. C'est surtout au Japon que des études fondamentales sur la plasticité des siliciures [40] ont été menées. Une application possible des siliciures réfractaires est leur utilisation comme matériaux de structure à haute température et c'est ce qui a motivé ces études menées sur monocristaux. Des études systématiques dans les siliciures tétragonaux $\mathrm{C} 11 \mathrm{~b}\left(\mathrm{MoSi}_{2}\right.$ and $\left.\mathrm{WSi}_{2}\right)$, hexagonaux $\mathrm{C} 40\left(\mathrm{CrSi}_{2}, \mathrm{NbSi}_{2}, \mathrm{TaSi}_{2}\right)$ et orthorhombique C54 $\left(\mathrm{TiSi}_{2}\right)$ ont été menées pour identifier les systèmes de glissement actifs et la dépendance en température de la contrainte critique résolue. La contrainte critique résolue dépend très fortement de la température, ce qui indique l'existence de vallées de Peierls profondes. Ceci est en accord avec le caractère partiellement covalent de la liaison puisque l'énergie de Peierls est généralement plus élevée [41] dans les cristaux covalents que dans les métaux. A basse température (typiquement en dessous de $500^{\circ} \mathrm{C}$ ) peu de systèmes de glissement sont actifs. En particulier dans les siliciures hexagonaux C40 seul le plan basal est actif. On s'attend donc à ce que la texture influence fortement les propriétés mécaniques des films de siliciures polycristallins. Par exemple un film hexagonal orienté (00.1) ne devrait pas se déformer plastiquement à basse température. Plus généralement des variations importantes [42] de contraintes de grain à grain sont attendues. La plasticité de $\mathrm{CoSi}_{2}$ [43] a aussi été étudiée. $\mathrm{CoSi}_{2}$ cristallise dans la structure cubique $\mathrm{C1}$ $\left(\mathrm{CaF}_{2}\right)$. Le système de glissement primaire [43] entre la température ambiante et $1000^{\circ} \mathrm{C}$ est $\{001\}\langle 100\rangle$, alors que $\{111\}\langle 110\rangle$ et $\{110\}\langle 110\rangle$ sont des systèmes de glissement secondaires qui ne sont actifs qu'au dessus de $500^{\circ} \mathrm{C}$. La variation rapide de la contrainte critique résolue avec la température indique là encore un mécanisme de Peierls. $\mathrm{CoSi}_{2}$ semble être le seul composé $\mathrm{C} 1$ pour lequel le système de glissement primaire soit $\{001\}\langle 100\rangle$. Les fluorures ioniques tels que $\mathrm{CaF}_{2}, \mathrm{BaF}_{2}$ ou $\mathrm{SrF}_{2}$ ont un système primaire du type $\{001\}\langle 110\rangle$ et les hydrures métalliques $\mathrm{C} 1$ ont un système primaire $\{111\}\langle 110\rangle$ comme les métaux cubiques à faces centrées. Les films épitaxiés de $\mathrm{CoSi}_{2}$ ont été très étudiés dans les années 1980 [44, 45] en vue de leur application dans des transistors à base métallique. Dans les couches de $\mathrm{CoSi}_{2}(111)$ le seul système de glissement qui a été observé $[46,47]$ est $\{111\}\langle 110\rangle$. Dans la mesure où ces films ont été formés à haute température cette observation n'est pas contradictoire avec ce qui a été observé dans les monocristaux massifs.

Les films de siliciures utilisés en microélectronique ont aujourd'hui des épaisseurs très faibles, de l'ordre de $20 \mathrm{~nm}$. Il est donc nécessaire de prendre en compte l'influence de la taille sur les propriétés mécaniques de ces films. Il est maintenant bien établi [18-20] que les films minces de métaux simples $(\mathrm{Al}, \mathrm{Cu}, \mathrm{Ag}, \mathrm{Au})$ ont des limites élastiques très supérieures aux métaux massifs. A titre d'exemple un film de Cu d'épaisseur $500 \mathrm{~nm}$ a une limite élastique [20] de l'ordre de 400-500 MPa. La plupart des auteurs s'accordent sur une variation de la limite élastique en $1 / \mathrm{h}$ où $\mathrm{h}$ est l'épaisseur. Une variation donc différente de la loi de Hall et Petch qui stipule que la limite élastique varie avec l'inverse de la racine carrée de la taille de grains. Des expériences systématiques [47, 48] ont permis de séparer l'influence de la taille des grains de celle de l'épaisseur. L'influence de l'épaisseur sur la limite élastique est décrit par un modèle de type Orowan souvent nommé «canalisation » des dislocations $[19,49]$.

Dans le paragraphe précédent on a considéré une relaxation plastique du film de siliciure, par glissement de dislocations. Lorsque la température augmente le fluage diffusionnel devient un mécanisme important de relaxation de la contrainte. On prédit que le fluage puisse se produire à d'autant plus basse température que les films sont minces. Gao [50] a modélisé le fluage diffusionnel confiné dans des films de $\mathrm{Cu}$. Dans le cas des films de siliciures la relaxation par un mécanisme diffusionnel requiert le mouvement des deux espèces atomiques (le métal et le silicium). On peut donc s'attendre à ce que l'énergie d'activation caractéristique de la relaxation (liée à l'espèce qui diffuse le plus lentement) soit supérieure à l'énergie d'activation caractéristique de la croissance (liée à l'espèce qui diffuse le plus vite). 


\section{CONCLUSIONS}

Les siliciures de métaux de transition à caractère métallique ont été introduits en microélectronique au début des années 1980. Les conditions imposées par la taille toujours plus petite des transistors ont contraint les technologues à changer régulièrement d'intermétallique et arriver aujourd'hui à l'utilisation de NiSi. Les siliciures sont formés par diffusion réactive entre un film mince de métal et le silicium. Cette réaction en phase solide donne lieu à un développement considérable de contraintes. Au-delà de l'intérêt fondamental à comprendre les mécanismes responsables de l'établissement de ces contraintes, la description quantitative des déformations induites dans le silicium est nécessaire pour contrôler la mobilité des porteurs de charge. Beaucoup reste à faire pour décrire précisément le développement des contraintes résultant de la formation des siliciures. En particulier la connaissance des propriétés mécaniques des couches minces de siliciure est absolument nécessaire.

\section{Remerciements}

Les travaux décrits dans cet article viennent en partie des travaux de thèse de Makram Megdiche et Christian Rivero. Les collaborations avec Patrice Gergaud, Bernard Chenevier et Marc Gailhanou ont été très fructueuses. Qu'ils en soient ici remerciés. Je dédie cet article à mon ami François d'Heurle qui nous a quittés en octobre 2007 et qui a été un pionnier dans l'étude des films minces de siliciures.

\section{Références}

[1] Nicolet M.A. et and Lau S.S. "Formation and Characterization of Transition-Metal Silicides", in VLSI Electronics, Microstructure Science. Vol 6 (Academic Press, NY, 1983).

[2] Miglio L. and d'Heurle F.M., in Silicides : Fundamentals and Applications (Proceedings of the $16^{\text {th }}$ course of the international school of solid state physics, Erice, Italy, 2000).

[3] Kilby J., IEEE Trans. Electron Dev. 23 (1976) 648.

[4] www.itrs.net

[5] Crowder B. and Zirinski S., IEEE Trans. Electron Dev. 26 (1979) 369

[6] Nava F., Tu K., Borghesi A., Guizzetti G., Laborde O., Thomas O., Madar R., Sénateur J.P. and Bisi O., Materials Science Reports 9 (1993) 141.

[7] Maex K. et and Van Rossum M., in Properties of Metal Silicides, Mat. Sci. Eng. R11 (1993) 53.

[8] d'Heurle F.M. and Gas P., J. Mater. Res. 1 (1986) 205.

[9] d'Heurle F.M., J. Mater. Res. 3 (1988) 167.

[10] Gosele U. and Tu K., J. Appl. Phys. 53 (1982) 3252.

[11] Thomas O., Delage S., d'Heurle F.M. and Scilla G., Appl. Phys. Lett. 54 (1989) 228.

[12] d'Heurle F.M. and Thomas O., Defect and diffusion forum, 129-130 (1996) 137.

[13] Welser J., Hoyt J. and Gibbons, J. IEEE Electron Device Lett. 15 (1994) 100.

[14] Rhines F. and Wolf J., Metall. Trans. 1 (1970) 701.

[15] Cahn J. and Larche F., Acta metall. 33 (1985) 331.

[16] Stephenson G.B., Acta metall. 36 (1988) 2663.

[17] Krishnamurty R. and Srolovitz D., Acta Mat. 51 (2003) 2171.

[18] Kuan T.S. and Murakami M., Metall. Trans. A 13A (1982) 383.

[19] Nix W.D., Metall. Trans. A 20 (1989) 2217.

[20] Arzt E., Prog. Mat. Sci. 46 (2001) 283.

[21] Stoney G.G., Proc. R. Soc. London Ser. A 82 (1909) 172.

[22] Gardner et D.S. and Flinn P.A., IEEE Trans. Electron. Dev. 35 (1988) 2160.

[23] Cicariello J., Poize S. and Gas, P. J. Appl. Phys. 67 (1990) 3315.

[24] Gas P. and d'Heurle F.M., Mat. Res. Symp. Proc. 402 (1996) 39.

[25] Gergaud P., Megdiche M., Thomas O. and Chenevier B., Appl Phys. Lett. 83 (2003) 1334. 
[26] Coble R.L., J. Appl. Phys. 34 (1963) 1679.

[27] Zhang S.L. and d'Heurle F.M., Thin Solid Films 213 (1992) 34.

[28] Cacho F., Cailletaud G., Rivero C., Gergaud P., Thomas O. and Jaouen H., Mat. Sci. Eng. B 135 (2006) 95.

[29] Guénin G., Ignat et M. and Thomas O., J. Appl. Phys. 68 (1990) 6515.

[30] Malegori G. and Miglio L., Phys. Rev. B 48 (1993) 9223.

[31] Hellwege K. and Hellwege A., Landolt Börnstein GIII, Vol. 11, Springer Verlag, Berlin (1979).

[32] Brantley W., J. Appl. Phys. 44 (1973) 534.

[33] Simmons G. and Wang H., in Single crystal elastic constants and calculated aggregate properties: a handbook (MIT press, Cambridge Mass. 1971).

[34] Wilson D.F. and Cavin O.B., Scripta Metall. Mater. 26 (1992) 85.

[35] Detavernier C., Lavoie C. and d'Heurle F., J. Appl. Phys. 93 (2003) 2510.

[36] Connétable D., communication privée (2008).

[37] Rivero C., PhD thesis Université Paul Cézanne, 2005.

[38] Thomas O. and Kristallogr Z., Suppl. 26 (2007) 81.

[39] Tersoff J. and Hamann D.R., Phys. Rev. B 28 (1983) 1168.

[40] Ito K., Moriwaki M., Nakamoto T.H., Inui M. and Yamaguchi, Mat. Sci. Eng. A 233 (1997) 33.

[41] Hirth J.-P. and Lothe J., in Theory of Dislocations (John Wiley \& Sons, 1982).

[42] Sanchez J.E. Jr. and Arzt E., Scripta Metall. Mater. 27 (1992) 285.

[43] Ito K., Inui H., Hirano T. and Yamaguchi M., Acta Metall. Mater. 42 (1994) 1261.

[44] Rosencher E., Delage S., Campidelli Y. and Arnaud d'Avitaya F., Electron. Lett. 20 (1984) 762.

[45] Hensel J.-C., Levi A.F., Tung R.T. and Gibson J.M., Appl. Phys. Lett. 49 (1986) 522.

[46] Bulle-Lieuwma C.W., Vandenhoudt D.E., Henz J., Onda N. and von Känel H., J. Appl. Phys. 73 (1993) 3220.

[47] Venkatraman R. and Bravman J.C., J. Mater. Res. 7 (1992) 2040.

[48] Doerner M.F., Gardner D.S. and Nix W.D., J. Mater. Res. 1 (1986) 845.

[49] Freund L., J. Appl. Mech. 54 (1987) 553.

[50] Gao H., Zhang L., Nix W., Thompson C. and Arzt E., Acta Mater. 47 (1999) 2865. 